\title{
Hypoglycaemia in diabetes mellitus - protecting the brain
}

\author{
S.A . A miel \\ Department of Medicine, King's College School of Medicine and Dentistry, London, UK
}

Summary The human brain generally uses glucose as its principal metabolic fuel but cerebral metabolism and function during hypoglycaemia can be supported by a variety of substrates. Different brain regions may vary in this. The brain's ability to sense a falling blood glucose concentration and initiate a protective response varies according to prior glycaemic experience. The brain's ability to use non-glucose fuels may ultimately be of therapeutic use. Meanwhile, avoidance of even moderate hypoglycaemia during normal clinical treatment of diabetes mellitus leads to restoration of protective symptomatic responses to early hypoglycaemia, without necessarily causing a deterioration in overall glycaemic control. [Diabetologia (1997) 40: S 62-S68]

Keywords Hypoglycaemia, counterregulation, diabetes mellitus, brain metabolism.

\section{Presentation and consequences of hypoglycaemia}

The principal danger of hypoglycaemia is the associated loss of brain function. Under normal circumstances, the brain is almost exclusively dependent on glucose to fuel its metabolism and function and, since its glucose stores are negligible, normal brain function relies upon an adequate supply of glucose from its blood supply.

In animal models, prolonged very profound hypoglycaemia eventually results in neurone death (compared with ischaemia, which results in non-selective loss of all brain cell types) [1]. In clinical practice, apparent full recovery is the rule even from severe episodes of hypoglycaemia. Permanent impairment of brain function is seen only after the most severe episodes, usually associated with massive insulin overdose, often either deliberate or malicious [2]. In general, the loss of brain function due to hypoglycaemia presents in any degree from mild confusion or

Corresponding author: Professor S. A. Amiel, Department of Medicine, King's College School of Medicine and Dentistry, Bessemer Road, London SE5 9PJ, UK

A bbreviations: NEFA, Non-esterified fatty acid; IDDM, insulin-dependent diabetes mellitus. disinhibition to coma and full recovery apparently occurs within an hour of restoration of normoglycaemia [3]. There are concerns that recurrent episodes of hypoglycaemia-associated coma may result in permanent damage to cortical (intellectual) function although in adults the data are inconclusive, with some studies suggesting an acceleration of the age-related decline in performance on IQ testing [4], while others relate this to associated diabetic neuropathy [5]. Prospective studies of people who have been at particular risk for recurrent severe hypoglycaemia show no effect on long-term cognitive function $[6,7]$. There is evidence of permanent impairment of IQ after recurrent hypoglycaemic episodes occurring before the age of 5 years (i.e. affecting the developing brain) [8]. (For a review of the possible long-term effects of recurrent hypoglycaemia, see [9]).

\section{P rotective mechanisms against severe hypoglycaemia}

In health, severe hypoglycaemia (now defined as the state in which the patient is unable to self-treat because of impaired function and has to be treated by someone else [10]), does not occur because of the efficient systems the body has to detect and deal with 
a falling blood glucose concentration. Thus, in fasting or in vigorous exercise, two circumstances under which circulating blood glucose levels could potentially fall, mechanisms of counterregulation are activated, which act to restore blood glucose levels. The mechanisms of counterregulation include: 1) reduction in endogenous insulin secretion; 2) stimulation of pancreatic glucagon secretion; 3 ) activation of the sympathetic nervous system; 4) stimulation of adrenaline, cortisol, growth hormone and other hormones such as pancreatic polypeptide; and 5) a symptom complex associated with the neurohumoral responses that the subject can learn to recognise as a 'warning' and which also stimulates the subject to eat.

These mechanisms stimulate hepatic glucose production, either directly (1 to 4 ) or indirectly, by providing substrates for gluconeogenesis (3 and 4); reducing the rate of utilisation of glucose by peripheral tissues such as muscle and fat (1,3 and 4$)$ and by providing new glucose (5).

\section{D efects in counterregulation in diabetes}

In diabetes, the mechanisms of counterregulation are impaired. The ability to adjust insulin levels spontaneously is lost if sulphonylureas or exogenous insulin are administered; the ability to secrete glucagon in response to hypoglycaemia is lost or least in insulin dependent patients [11] and in some patients, the ability to mount an autonomic and adrenergic response may also be impaired [11-13]. Loss of the efficiency of these mechanisms increases the risk not only of hypoglycaemia per se but also of severe episodes with clinically significant cognitive dysfunction.

Traditionally, impairments in counterregulation seen in some patients with diabetes is thought to be due to increasing disease duration and in some cases to the chronic complications of diabetes especially autonomic neuropathy, but this may not be the whole explanation. Classic autonomic neuropathy does not segregate with loss of symptomatic responses to hypoglycaemia [14], perhaps because the body becomes more sensitive to the diminished counterregulatory hormone responses it is able to mount [15]. Although loss of subjective awareness of hypoglycaemia is undoubtedly more common with increasing disease duration [16], it can occur early in the course of diabetes [17] and may be reversible in all cases [18].

Onset of counterregulation is likely to be a brain function. There is good evidence that the regions of the body that sense hypoglycaemia and initiate the counterregulatory responses are located in the brain $[19,20]$. There have been suggestions that hepatic glucose sensing may be equally or more important $[21,22]$, but the most recent investigations confirm the primacy of the brain [23]. The precise localisation of the putative glucose sensor is discussed elsewhere
Table 1. Potential fuels for cerebral metabolism

Glucose

Ketone bodies

Lactate

Fatty acids

Glycerol

Amino acids

[24]. Thus, both the onset of counterregulatory hormone responses and the onset of impaired performance in tests of cognitive function are reflections of how well the brain is functioning during a progressive fall in blood glucose. There is compelling evidence, discussed in more detail elsewhere [25], that a fall in the rate of cerebral glucose uptake is associated with or precedes both responses to hypoglycaemia [26].

\section{B rain metabolism and hypoglycaemia}

Although traditionally assumed to be an obligate glucose consumer, the brain contains the enzyme systems necessary for the metabolism of a range of substrates (Table 1). There is no prime facie reason why brain tissue should not therefore use a range of metabolic fuels to maintain its metabolism and function, particularly during times of glucose lack. One of the first substrates to be examined was ketones. In a now famous study by Owen and colleagues [27], negative ketone balance across the brain was observed in three morbidly obese individuals after 6 weeks of near starvation dieting. The brain in these circumstances had become a net consumer of ketone bodies. These data were interpreted to suggest that after a period of adaptation the human brain could convert to the use of ketones as a metabolic fuel. These observations received support from clinical observations that hypoglycaemia appeared to be better tolerated in persons after prolonged fast [28] but the idea remained that a period (unspecified) of adaptation was required for the brain to switch on the necessary enzyme systems. This was despite an elegant study in rodents [29] that demonstrated equal evidence of brain ketone utilisation in rats made acutely hyperketonaemic by ketone infusion as in animals made subacutely ketonaemic by prolonged fasting.

We induced hypoglycaemia in healthy volunteers in the presence or absence of an infusion of beta-hydroxybutyrate. Raising beta-hydroxybutyrate concentration tenfold during controlled stepped reduction in plasma glucose levels from 5 to $2.2 \mathrm{mmol} / \mathrm{l} \mathrm{de}-$ layed the onset and diminished the magnitude of the adrenaline responses, and was better tolerated [30]. These data were compatible with the hypothesis that the metabolism and therefore normal function (i.e. not activated) of the glucose sensor could be supported during hypoglycaemia by the non-glucose 


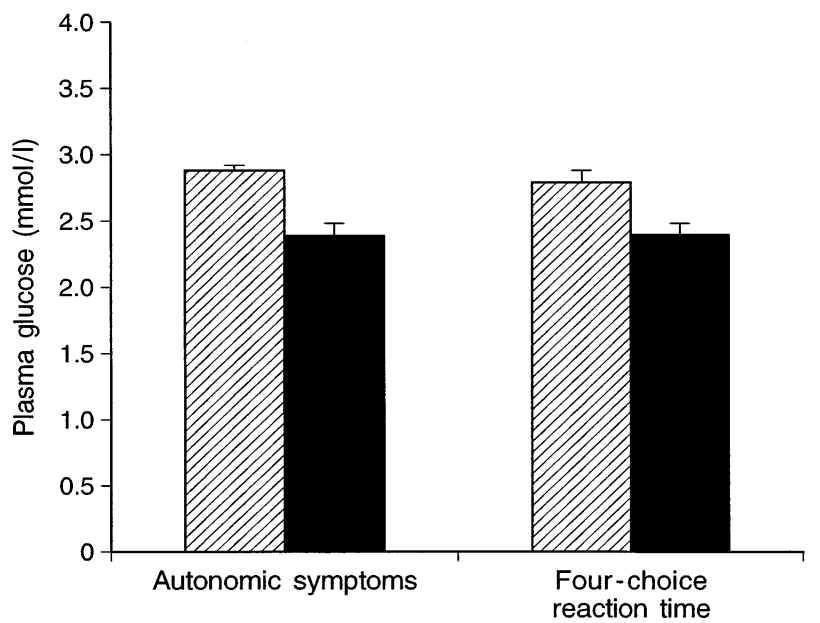

Fig. 1. Effect of lactate on glucose thresholds for symptomatic awareness and cognitive dysfunction during controlled stepped hypoglycaemia in healthy male volunteers. Control study $\mathbb{Z}$; in the presence of lactate $\square . p<0.005$ for differences between studies. Adapted from [33], with permission

metabolic fuel beta-hydroxybutyrate. Cognitive function was not measured in that study, but in a more recent but similar study, with a rather larger dose of beta-hydroxybutyrate, a delay in the onset of cognitive dysfunction was also demonstrable [31], thus confirming that some effect of the ketone bodies was cerebral, even if the detection of hypoglycaemia and onset of counterregulation were not.

These studies provide an explanation for the clinical presentation of ketotic hypoglycaemic states in childhood [32]; however, except in the special circumstances of inborn errors of metabolism, ketones are unlikely to provide a useful therapeutic manoeuvre for protecting cerebral function during hypoglycaemia in pharmacological treatment of diabetes, although ketogenic diets have been used in the management of children with some forms of intractable epilepsy, perhaps ameliorating a problem of glucose uptake. However, in insulin-induced hypoglycaemia as encountered in the treatment of diabetes, circulating ketone levels are suppressed by insulin. Another metabolic fuel with some potential for supplying the brain's metabolic needs is lactate. We recently demonstrated that infusion of sodium lactate, raising the circulating lactate levels to about $2.4 \mathrm{mmol} / \mathrm{l}$ was not only able to lower the plasma glucose level at which hormonal and symptomatic response to hypoglycaemia began but was also able to lower the glucose level at which the onset of cognitive dysfunction (in this case measured as a slowing or loss of accuracy in a four-choice reaction time test) began, again during controlled progressive step-wise hypoglycaemia in healthy male volunteers [33]. These results are shown in Figure 1 and have since been reproduced by others [31], although reduction of the lactate (and fatty acids) during hypoglycaemia in one study did not affect counterregulatory responses [34].
R egional variation in the metabolic capacity of the brain

As with the ketone studies, infusion of lactate is not an immediately useful therapeutic tool for the prevention of higher brain dysfunction during hypoglycaemia in the daily life of people with treated diabetes. It does offer the potential for developing such tools. Meanwhile these studies allow us to learn more about the metabolism of the human brain both regionally and locally. The possibility of regional differences in cerebral metabolic potential is beginning to assume clinical significance in the aetiology of hypoglycaemia unawareness in insulin-treated diabetes. This syndrome is associated with impairment of the normal counterregulatory responses to hypoglycaemia, such that the hormonal responses to a given hypoglycaemic challenge are diminished. As mentioned above, it occurs in patients with long-standing diabetes, but defects in counterregulatory responses to hypoglycaemia are also seen in people with very tightly controlled diabetes [35] and have been induced during intensified insulin therapy in people with relatively short diabetes duration [17]. The principal defect appears to be a lowering of the plasma glucose level initiating the counterregulatory response, associated with a delay in the onset of the symptoms of hypoglycaemia [36]. This argues that the cells of the cerebral glucose sensor adapt to some circumstance of intensified insulin therapy (or to their blood supply) such that they can better maintain their metabolism and function during subsequent hypoglycaemia. If all the brain tissue could so adapt, the syndromes of acute hypoglycaemia would be the same in intensively- as in conventionally-treated diabetes, albeit occurring at lower glucose levels but with the same relationship of symptomatic and protective responses preceding (and therefore protecting against) the more profound hypoglycaemia needed to cause significant cognitive impairment. Indeed, there is evidence to support this view, with deterioration of performance on multiple cognitive function tests (brought to a single value as a summed Z-score) [17]; the Stroop test and finger tapping all apparently occurring at a lower glucose level in hypoglycaemia unaware subjects when tested formally [24, 37]. Nevertheless, the clinical syndromes of hypoglycaemia unawareness are clearly associated with increased risk of severe hypoglycaemia, especially in intensified insulin therapy, where both hypoglycaemia without symptoms and severe hypoglycaemia (where cortical brain function is so disturbed that the victim is unable to self-treat and requires resuscitation by someone else) are three times more frequent than in patients receiving conventional therapy $[6,10,38]$. Our own data, using a single test of cognitive function (fourchoice reaction time), have repeatedly noted a difference in the way the glucose sensing areas and the 


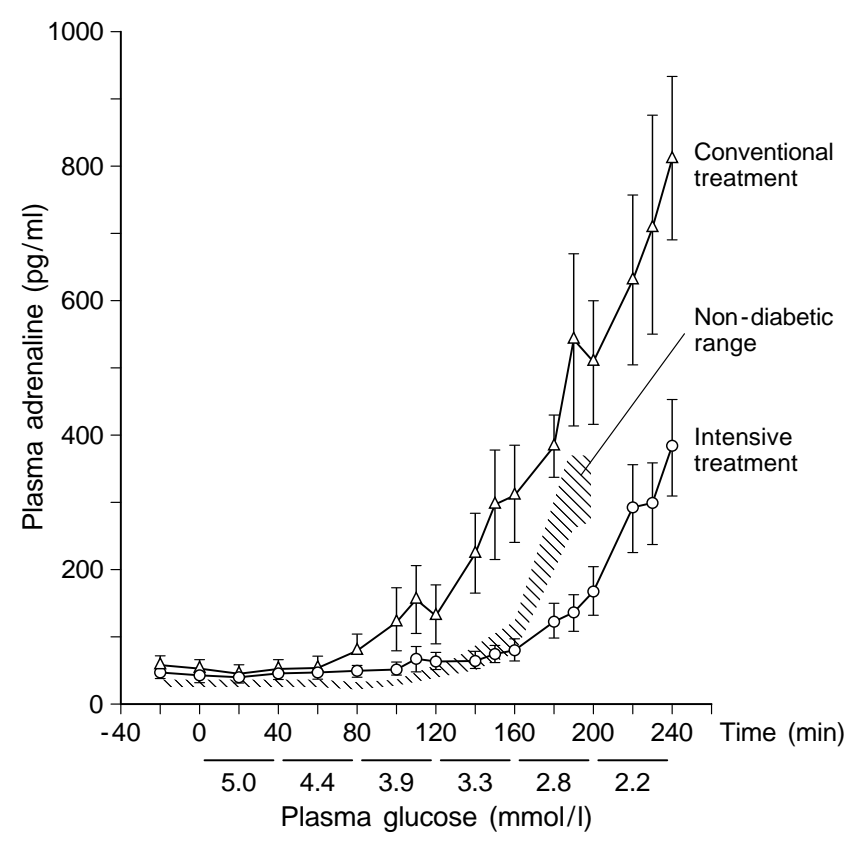

Fig. 2. Effect of intensified insulin therapy on adrenaline responses to hypoglycaemia. Adapted from [36] with permission

cognitive areas of the brain behave in hypoglycaemia unaware people, with no evidence of a lowering of the glucose level for cognitive dysfunction in hypoglycaemia despite marked lowering of the glucose level for counterregulatory hormone and symptom responses [18, 39]. Similarly, Widom and Simonson [40] were unable to find an effect of glycaemic control on glucose thresholds for deterioration in performance of individual cognitive function tests. These data provide a mechanism for the increased risk of both asymptomatic and severe hypoglycaemia in intensively treated diabetes and also provide evidence of regional differences in the way brain tissue, and/ or its circulatory responses, adapts to different degrees of glycaemic control. Regional differences even within the cerebral cortex might also explain the discrepancies between our data using the fourchoice reaction time test and those of others using other cognitive function tests, which use different cerebral pathways. Further evidence of a difference in regional cerebral metabolic capacity is provided by preliminary evidence from an on-going study of the brain's ability to use non-esterified fatty acids (NEFA) during hypoglycaemia. Elevation of NEFA (as well as triglyceride and glycerol) may reduce catecholamine and symptom responses to hypoglycaemia but does not appear to be able to support cognitive function [41]. Thus, there is accumulating evidence of regional variations in cerebral metabolism and function, which may eventually be exploitable in devising therapies to protect higher brain function during hypoglycaemia and provide defence against severe hypoglycaemia without warning. Recent studies with positron emission tomography suggest regional

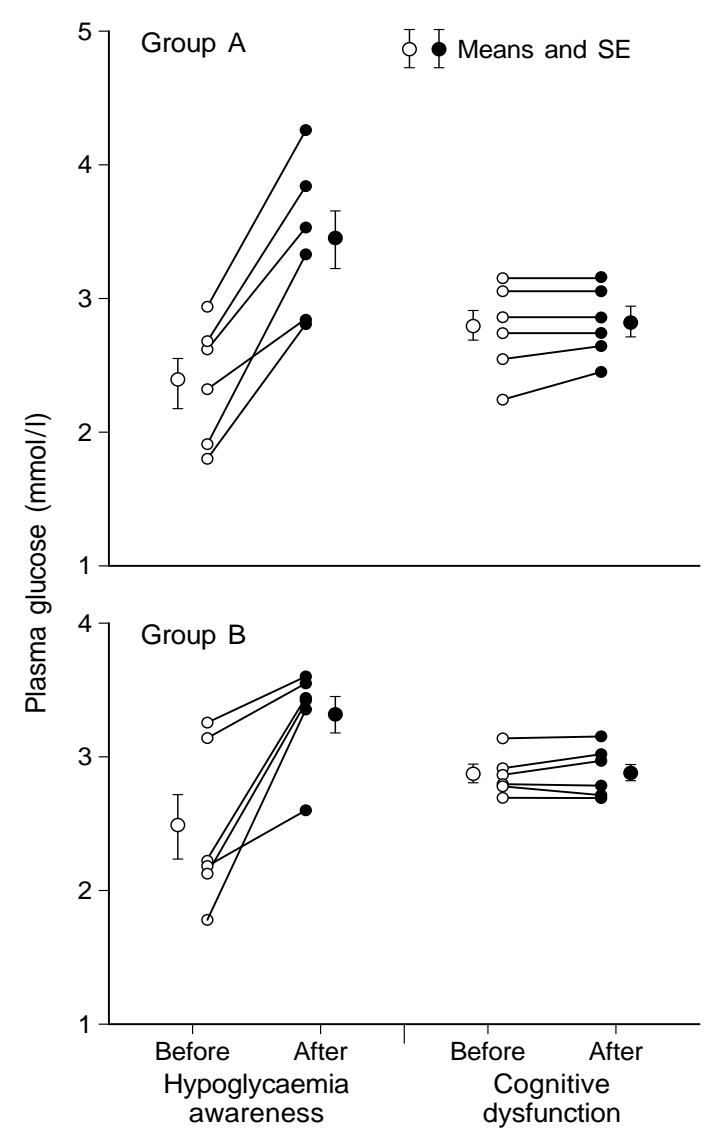

Fig. 3. Normalisation of glucose thresholds for subjective awareness (with no effect on thresholds for cognitive dysfunction) by avoidance of recurrent clinical mild-moderate hypoglycaemia in 6 tightly controlled hypoglycaemia-prone IDDM patients. Adapted from [18]

variations of glucose metabolism itself in human brain, which may be explored in an attempt to understand the functional responses of the brain to hypoglycaemia and how they adapt in clinical situations [42].

The role of amino acids in human cerebral metabolism remains to be fully investigated. Cryer et al. [43] recently suggested that including alanine in the pre-bedtime snack may help prevent hypoglycaemia at night. While this may be because the amino acid provides a stimulus for glucagon secretion or a fuel for gluconeogenesis, it is also possible the amino acids are able to support cerebral function during hypoglycaemia directly - a possibility currently under investigation in our laboratory.

\section{R estoration of the defective counterregulation of insulin-dependent diabetes (ID D M )}

The elevation of non-glucose substrates to support cerebral metabolism and function during hypoglycaemia remains an exciting avenue for further exploration. Meanwhile, there is already evidence that the 
metabolism of the human brain can be manipulated to protect against episodes of severe hypoglycaemia with cognitive dysfunction in patients under treatment for diabetes.

The defective symptomatic and counterregulatory responses to hypoglycaemia that can be induced in diabetic patients during intensification of insulin therapy have already been discussed. Such patients show a lowering of the plasma glucose level at which the hormonal and symptomatic responses to hypoglycaemia occur. A similar defect can also be induced acutely in non-diabetic healthy volunteers by recent prior mild hypoglycaemia ( 3 or $2.8 \mathrm{mmol} / \mathrm{l}$ ) [44] and in people with diabetes [45], leading to suggestions that recurrent mild hypoglycaemia experienced during the drive for normoglycaemia in diabetes (as exemplified by intensified therapy) underlies the defects seen more chronically in intensively treated IDDM. One of the contenders for this adaptation is an upregulation of glucose transporters in brain exposed to chronic or recurrent hypoglycaemia and recently there has been a demonstration of increased mRNA for GLUT1 transporters (the endothelial transporter presumably responsible for glucose crossing the blood-brain barrier) in an animal model of recurrent hypoglycaemia, while the neuronal glucose transporter, GLUT3, remains apparently unaffected [46]. If this hypothesis is correct, then avoidance of even minor degrees of hypoglycaemia in clinical life may prevent the development of defective glucose counterregulation in diabetes or even be able to restore it in patients with hypoglycaemia unawareness.

We studied two groups of people with long-standing diabetes each with a history of recurrent severe hypoglycaemia [18]. The first group were typical of hypoglycaemia-prone tightly controlled diabetic patients with $\mathrm{HbA}_{1 \mathrm{c}}$ levels near the normal range, but the other group were classified as in poor control, with high glycated haemoglobin levels and generally high blood glucose readings, which nevertheless occasionally and often unexpectedly fell into severe hypoglycaemia. Their responses to a formal hypoglycaemic challenge were tested under laboratory conditions and were found to be defective, starting late (i.e. at lower plasma glucose levels than would be seen normally) and with a reversed hierarchy of responses, with impaired cognition (four-choice reaction time) occurring at a higher plasma glucose level than symptomatic and protective responses. Each patient then embarked on a regimen of diabetes management that aimed only at avoiding blood glucose readings below $3.5 \mathrm{mmol} / \mathrm{l}$. Re-study was not performed until after 3 weeks of no documentation of blood glucose values below $3 \mathrm{mmol} / \mathrm{l}$ (on intensive home monitoring including regular nocturnal checks). This was achieved by scrupulous attention to the timing of injections, and meal and snack ingestion, and full compensation of insulin dose for exercise in the subjects with well-controlled diabetes, with conversion to basal-bolus regimens for the patients in poor metabolic control where needed. Parenthetically, the most important 'error' in the patients' initial regimens was the lack of postprandial snacks and the failure to appreciate the duration of the hypoglycaemic effects of sustained or vigorous exercise. After an average of 4 months' avoidance of even mild clinical hypoglycaemia, a dramatic restoration of the catecholamine, growth hormone and cortisol responses was seen, together with restoration of subjective awareness of hypoglycaemia and, perhaps most important, a restoration of the protective hierarchy of hormonal and symptomatic responses occurring before the onset of cognitive dysfunction during progressive controlled hypoglycaemia. The avoidance of hypoglycaemia was not associated with any deterioration of average glycaemic control. $\mathrm{HbA}_{1 \mathrm{c}}$ did not alter during the study in either group. Similar results had been found in patients with short-duration diabetes and recurrent hypoglycaemia, although with less complete restoration perhaps because of less complete elimination of preceding hypoglycaemia [17], but the Lancet study [18] not only showed the possibility of restoration of hypoglycaemia awareness in long-duration disease, but also in patients with generally poor control, suggesting a common aetiology and a common potential cure for all cases of hypoglycaemia without warning.

What clinical messages can we learn from these data? Perhaps the most important is that good diabetes control should include a lower as well as a higher limit to the desirable glycaemic range. Defects in counterregulation can be induced in healthy subjects (albeit acutely) by lowering blood glucose transiently to $3 \mathrm{mmol} / \mathrm{l}$, and such glucose levels should not be considered acceptable in the management of diabetes. Relaxation of diabetic control may sometimes be necessary in a patient who has developed hypoglycaemia unawareness but is not absolutely necessary if the patient can avoid blood glucose concentrations below the non-diabetic range (e.g. less than $4 \mathrm{mmol} / \mathrm{l}$ ). With the same degree of effort as currently put into avoiding blood glucose concentrations above the non-diabetic range, we may then be able to achieve truly 'good' glycaemic control. One of the major problems is the lack of exogenous insulins that can mimic either the very high but very transient prandial bursts of endogenous insulin from the healthy pancreas, or the constant low background of insulin excreted overnight, although some of the new insulin analogues offer hope for the future. Meanwhile an understanding of the action profiles of insulin and the interactions that eating, exercise and even alcohol can have on its effects can go a considerable way towards protecting against loss of counterregulatory responses and severe hypoglycaemia in the most intensively treated diabetes. 


\section{References}

1. Kristian T, Gido G, Siejo B (1995) The influence of acidosis on hypoglycemia brain damage. J Cerebr Blood Flow Metab 15: 78-87

2. Lins PE, Adamson U (1993) Neurological manifestations of hypoglycaemia. In: BM Frier, M Fisher (eds) Hypoglycaemia and diabetes. Clinical and physiological aspects. Edward Arnold London p 348

3. Herold KC, Polonsky KS, Cohen RM, Levy J, Douglas F (1985) Variable deterioration in cortical function during insulin-induced hypoglycemia. Diabetes 34: 677-685

4. Langhan SJ, Deary IJ, Hepburn DA, Frier BM (1991) Cumulative cognitive impairment following recurrent hypoglycaemia in adult patients. Diabetologia 34: 337-344

5. Ryan CM, Williams TM, Finegold DN, Orchard TJ (1993) Cognitive dysfunction in adults with type 1 diabetes mellitus of long duration: effects of recurrent hypoglycaemia and other chronic complications. Diabetologia 36: 329-334

6. Diabetes Control and Complications Research Group (1993) The effect of intensive treatment of diabetes on the development and progression of long-term complications in insulin dependent diabetes mellitus. N Engl J Med 329: 977-986

7. Reichard P, Britz A, Rosenqvist U (1991) Intensifed conventional insulin treatment and neuropsychological impairment. BMJ 303: 1439-1442

8. Rovet JE, Ehrlich RM, Hoppe M (1987) Intellectual deficits associated with early onset of insulin dependent diabetes mellitus in children. Diabetes Care 10: 510-515

9. Gold AE, Deary IJ, Jones RW, O'Hare JP, Reckless JPD, Frier BM (1995) Severe deterioration in cognitive function and personality in five patients with long-standing diabetes: a complication of diabetes or a consequence of treatment. Diabet Med 11: 499-505

10. Diabetes Control and Complications Research Group (1991) Epidemiology of severe hypoglycaemia in the Diabetes Control and Complications Trial. Am J Med 90: 450-459

11. Bolli G, DeFeo P, Campugnucci P et al. (1983) Abnormal glucose counterregulation in insulin-dependent diabetes mellitus. Interaction of anti-insulin antibodies and impaired glucagon secretion. Diabetes 32: 134-141

12. Bolli GB, Dimitriadis GD, Pehling GB et al. (1984) Abnormal glucose counterregulation after subcutaneous insulin in insulin dependent diabetes mellitus. N Engl J Med 310: 1706-1711

13. Hoeldtke RD, Boden G, Shumna CR, Owen OE (1982) Reduced epinephrine secretion and hypoglycaemia unawareness in diabetic autonomic neuropathy. Ann Intern Med 73: 403-411

14. Ryder RJ, Owens DR, Hayes TM, Chatei MA, Bloom SR (1990) Unawareness of hypoglycaemia and inadequate hypoglycaemic counterregulation: no causal relationship with diabetic autonomic neuropathy. BMJ 301: 783-787

15. Hilsted J, Richter F, Madsbad S et al. (1987) Metabolic and cardiovascular responses to epinephrine in diabetic autonomic neuropathy. N Engl J Med 317: 421-426

16. Hepburn DA, Patrick AW, Eadington DW, Ewing DJ, Frier BM (1990) Unawareness of hypoglycaemia in insulin-treated diabetic patients: prevalence and relationship to autonomic neuropathy. Diabet Med 7: 711-717

17. Fanelli CG, Epifano L, Rambotti AM (1993) Meticulous prevention of hypoglycaemia normalizes glycemic thresholds and magnitude of most of the neuroendocrine responses to, and symptoms of, and cognitive function during hypoglycemia in intensively-treated patients with short-term IDDM. Diabetes 42: 1683-1689

18. Cranston I, Lomas J, Maran A, Macdonald IA, Amiel SA (1994) Restoration of hypoglycaemia awareness in patients with long duration insulin dependent diabetes mellitus and a history of hypoglycaemia without warning. Lancet 344 : 283-287

19. Biggers DW, Myers SR, Neal D et al. (1989) Role of the brain in counterregulation of insulin induced hypoglycaemia in dogs. Diabetes 38: 7-16

20. Frizzell RT, Jones EM, Davis SN et al. (1993) Counterregulation during hypoglycaemia is directed by widespread brain organs. Diabetes 42: 1253-1261

21. Donovan C, Halter JB, Bergman RN (1991) Importance of hepatic glucoreceptors in sympathoadrenal response to hypoglycemia. Diabetes 40: 155-158

22. Hamilton Wessler M, Donovan C, Halter J, Bergman R (1995) Liver glucosensors for hypoglycaemia responsive to rate of glucose fall. Diabetes 44 [Suppl 1]:111A (Abstract)

23. Davis S, Shavers C, Neal D, Allen E, Williams P (1995) Hepatic hypoglycaemia is unable to initiatiate counterregulation in conscious dogs. Diabetes 44 [Suppl 1]:3A (Abstract)

24. Maggs DG, Borg WP, During MJ, Sherwin RS (1997) Microdialysis techniques in the study of brain and skeletal muscle. Diabetologia 40: S75-S 82

25. Boyle PJ (1997) Alteration in brain glucose metabolism induced by hypoglycaemia in man. Diabetologia 40: S69-S 74

26. Boyle PJ, Nagy RJ, O'Connor AM, Kempers SF, Yeo RA, Qualls C (1994) Adaptation in brain glucose uptake following recurrent hypoglycemia. Proc Natl Acad Sci USA 91: 9352-9356

27. Owen OE, Morgan AP, Kemp HG, Sullivan JM, Herrara HG, Cahill GF (1967) Brain metabolism during fasting. J Clin Invest 46: 1590-1595

28. Drenick EJ, Alvarez LC, Tamasi GC (1972) Resistance to symptomatic insulin reactions after fasting. J Clin Invest 51: $2757-2762$

29. Fourest-Fontecave S, Adamson U, Lins PE, Ekblom B, Standhal C, Strand L (1987) Mental alertness during hypoglycaemia in normal man: the effect of 12 and 72 hours fasting. Diabet Metab 13: 405-410

30. Hawkins RA, Williamson DH, Krebs HA (1971) Ketone body utilization by adult and suckling rat brain in vivo. Biochemical J 122: 13-18

31. Amiel SA, Archibald HR, Chusney G, Williams AJK, Gale EAM (1991) Ketone infusion lowers hormonal responses to hypoglycaemia: evidence for acute cerebral utilization of a non glucose fuel. Clin Sci 81: 189-194

32. Veneman T, Mitrakou A, Mokan M, Cryer P, Gerich J (1994) Effect of hyperketonemia and hyperlacticacidemia on symptoms, cognitive dysfunction and counterregulatory hormone responses during hypoglycemia in normal humans. Diabetes 43: 1311-1317

33. Maran A, Cranston I, Lomas J, Macdonald IA, Amiel SA (1994) Protection by lactate of cerebral function during hypoglycaemia. Lancet 343: 16-20

34. Fanelli C, Di Vincenzo A, Modarelli F et al. (1993) Post-hypoglycaemic hyperketonaemia does not contribute to brain metabolism during insulin induced hypoglycaemia in humans. Diabetologia 36: 1191-1197

35. Amiel SA, Tamborlane WV, Simonson DC, Sherwin RS (1987) Defective glucose counterregulation after strict control of insulin-dependent diabetes mellitus. N Engl J Med 316: 1376-1383

36. Amiel SA, Sherwin RS, Simonson DC, Tamborlane WV (1988) Effect of intensive insulin therapy on glycemic 
thresholds for counterregulatory hormone release. Diabetes 37: 901-907

37. Boyle PJ, Kempers SF, O'Connor AM, Nagy R (1995) Brain glucose uptake and unawareness of hypoglycemia in patients with insulin dependent diabetes mellitus. New Engl J Med 333: 1726-1731

38. Lager I, Atvall S, Blohme G, Smith U (1986) Altered recognition of hypoglycaemic symptoms in type 1 diabetes during intensified control with continuous subcutaneous insulin infusion. Diabet Med 3: 322-325

39. Maran A, Lomas J, Macdonald IA, Amiel SA (1995) Lack of preservation of higher brain function during hypoglycaemia in patients with intensively treated diabetes mellitus. Diabetologia 38: 1412-1418

40. Widom B, Simonson DC (1990) Glycaemic control and neurophysiologic function during hypoglycaemia in patients with insulin dependent diabetes mellitus. Ann Intern Med 112: 904-912

41. Evans M, Lomas J, Matyka K, Cranston I, Macdonald IA, Amiel SA (1995) The effects of non-esterified fatty acid infusion on cerebral function during hypoglycaemia. Diabet Med 12 [Suppl 1]: S11-S12
42. Cranston I, Marsden PK, Evans M, Lomas J, Amiel SA (1996) Cerebral glucose utilisation - evidence for regional differences in rates of glucose transport vs phosphorylation. Diabet Med 13 [Suppl 3]: S34

43. Weithop BV, Cryer P (1992) Alanine and terbutaline in the treatment and prevention of hypoglycemia in IDDM. Diabetes 41 [Suppl 1]: 57A (Abstract)

44. Heller SR, Cryer PE (1991) Reduced neuroendocrine and symptomatic responses to subsequent hypoglycaemia in non-diabetic humans. Diabetes 40: 223-226

45. Dagogo Jack SE, Craft S, Cryer PE (1993) Hypoglycemia associated autonomic failure in insulin dependent diabetes mellitus. Recent antecedent hypoglycemia reduces autonomic responses to and symptoms of defense against subsequent hypoglycemia. J Clin Invest 91: 819-828

46. Kumagai AK, Kang Y-S, Boado RJ, Pardridge W (1995) In vivo up regulation of blood brain barrier to glucose transporter mRNA and protein in chronic hypoglycaemia. Diabetes 44 [Suppl 1]: 4A (Abstract) 